\section{Chancenfelder einer profilierten Diakonie}

\section{Urs Frey}

Was macht Soziale Arbeit in der Kirche aus und wie kann sie in der $\mathrm{Zu}-$ kunft erfolgreich sein? Vier Gedanken für eine profilierte, nicht konfessionsgebundene Diakonie: ${ }^{1}$

"Soziale Arbeit: wer hat sie erfunden? Wir - die Kirchel» So etwa könnte man sich einen Werbespot für die diakonische Arbeit vorstellen. Doch an den schweizerischen Ausbildungsstätten für Soziale Arbeit ist davon wenig mehr festzustellen. Manche Schulen haben zwar kirchliche Wurzeln. Aber etwa die Zürcher Schule (heute ZHAW Departement Soziale Arbeit) gibt sich seit ihren Ursprüngen 1908 betont säkular und Diakonie tritt im Curriculum nicht als eigenständiges Handlungsfeld in Erscheinung. Die Kirche ist einfach ein anerkannter Praxisort für die Studierenden. Immerhin schlägt der von der kantonalen Hochschule und der Evangelisch-reformierten Zürcher Landeskirche gemeinsam angebotene Weiterbildungskurs CAS Diakonie eine Brücke zwischen Staat und Kirche; einer Kirche notabene, die staatlich verfasst ist, sich daher als Volkskirche versteht und sich insbesondere im diakonischen Feld an Menschen zu richten hat, die den immer enger werdenden Kreis bekennender reformierter Christen sprengt. Diese Ausgangslage wirft die spannende Frage auf, was denn «Soziale Arbeit in der Kirche» - so lautet der Untertitel des besagten Lehrgangs - besonders macht.

Zunächst fällt auf, dass Sozialämter und kirchliche Sozialdienste die aktuellen sozialen Brennpunktthemen in der Schweiz ähnlich benennen. Die Grenzen des Wohlfahrtsstaates und wieder aufkommende versteckte

一

1 Dieser Beitrag erschien zuerst auf dem Fachportal der Konferenz Diakonie Schweiz, URL: https://www.diakonie.ch/chancenfelder-einer-profilierten-diakonie/ (abgerufen am 22.04.2021).

Jahrbuch Diakonie Schweiz 4 (2020/2021) - ISSN 2504-3994

Dieser Text ist lizenziert unter einer Creative Commons Namensnennung 4.0 International Lizenz (CC BY 4.0): (https://creativecommons.org/licenses/by/4.0/)
Armut; Wohnungsnot samt drohender Segregation und Gentrifizierung; der Verlust des gesellschaftlichen Zusammenhalts und damit drohender Einsamkeit; Alters- und Generationenfragen sowie Themen der Migration, wie Unter- und Überschichtung, Flucht und Asyl gehören dazu. Entsprechend lauten Integration, Inklusion, Kohäsion, Partizipation sowie die Mobilisierung von Zivilgesellschaftlichem hüben wie drüben schlagwortartig die Ziele, welche sich daraus ableiten lassen.

Doch verweist diese Zielkongruenz nicht auf eine eklatante Doppelspurigkeit? Hat es der moderne Staat nicht längst übernommen, die relevanten sozialen Probleme zu orten und in den Griff zu kriegen? Achtet er dabei nicht darauf, professionell geschultes Personal einzusetzen, das innerhalb klar reglementierter Bahnen arbeitet und im Dienste des Wohls des ganzen Volkes für einen ökonomischen Mitteleinsatz besorgt ist? Was bleibt da der Kirche vorbehalten? Darauf sind zwei Antworten denkbar, eine stolze und eine kleinlaute. Die stolze behauptet den Mehrwert diakonischer Sozialarbeit, welche die Hilfestellung um eine seelsorgerische, spirituelle und letztlich religiöse Komponente erweitert und somit das Eigentliche - oder Proprium - der Diakone ausmacht. Doch so einleuchtend diese Antwort auch ist, sie mag gerade jene nicht zufrieden stellen, die einer durch Steuergeld alimentierten Kirche zurückhaltend bis ablehnend gegenüberstehen, weil sie sich weltanschaulich-religiöse Einmischungen und Vermischungen kategorisch verbitten.

Bleibt die kleinlaute Antwort: Der Staat kann nicht alles leisten, die Kirche muss in die Lücke springen. Angesprochen ist somit die gut helvetische Subsidiarität, welche ihren Ursprung im nur schemenhaft vorhandenen Wohlfahrtsstaat des 19. Jahrhunderts hat. Gedacht wird hierbei vor allem an eine quantitative Entlastung, an die Substitution staatlicher Aufgaben durch kirchlich finanzierte oder in deren Rahmen freiwillig geleistete Arbeit. Im Zeichen von neoliberalen Rufen nach dem schlanken Staat klingt diese Formel aus dem vorletzten Jahrhundert auch in den Ohren eines säkular denkenden und auf sein Geld bedachten Steuerzahlers durchaus verlockend und modern. Das Angebot kommt jedoch dort an 
seine Grenzen, wo - wie heute der Fall - die Kirche qua Mitgliederschwund immer weniger Eigenmittel generiert und die diakonischen Leistungen zusehends über das Steuersubstrat juristischer Personen staatlich finanziert werden.

Im Folgenden wage ich daher abseits der beiden Pole vier Chancenfelder kirchlicher Sozialer Arbeit zu skizzieren, die den Gedanken der Subsidiarität jenseits der Substitution wohlfahrtsstaatlicher Aufgaben und auch jenseits des kirchlich-religiös definierten «Propriums» der Diakonie weiterspinnen. Zu verstehen sind sie nur als richtungsgebende Entwürfe und Denkimpulse und keinesfalls als ausgereifte Konzepte.

\section{Flexibilität und Agilität als professionelle Qualitäten pfle-}

gen

Die Professionalisierung ist schon fast eine Ur-Debatte der Sozialen Arbeit und sie wird gerade auch vor dem Hintergrund einer langen Emanzipation dieser Tätigkeit aus der voluntaristischen, unbezahlten und mithin wissenschaftlich unreflektierten Tradition nach wie vor heftig geführt. Dabei geht es bekanntlich durchaus darum, mit der Orientierung an den Menschenrechten eine eigenständige Position zu definieren und die eigene Tätigkeit und die Klientinnen und Klienten vor obrigkeitlichen Zumutungen und staatlichen Übergriffen zu schützen. Diese Professionalität ist ein Anspruch, den auch die diakonische Arbeit an sich selber stellen muss. Dazu gehört nicht zuletzt das Bewusstsein, dass die politische und ökonomische Realität Professionalität auch anders prägt. Leicht gerät sie ins Fahrwasser von Disziplinierung, Reglementierung und Ökonomisierung, welche allesamt dazu angetan sind, Handlungs- und Ermessensspielräume einzuengen.

Ein Chancenfeld der kirchlichen Sozialen Arbeit besteht daher darin, abseits der obrigkeitlichen Verwaltungslogik diese Spielräume zu erhalten, auszuloten oder gar auszuweiten. «Menschliches Mass statt Reglemente» die «Fünfe grad sein lassen», aber auch einfach «sich Zeit nehmen für den
Jahrbuch Diakonie Schweiz 4 (2020/2021) - ISSN 2504-3994
Menschen; ihm empathisch begegnen» wären handlungsleitende Losungen dazu. Das sind per se keine wissenschaftlichen Kategorien und gewiss heikel, wenn sie nolens volens zur Richtschnur genommen werden. Doch sie verweisen durchaus auf eine reflektierende und diskursive Kultur, wie sie als konstitutives Element Sozialer Arbeit seit jeher gepflegt wird. Es handelt sich hierbei aber auch um Arbeitsformen, die der kirchlichen Praxis jenseits der Verkündigung von Glaubensinhalten vertraut sind, ja recht eigentlich und hoffentlich ein Kulturmerkmal der Institution Kirche bilden.

Das ist nun allerdings keine Einladung zum selbstgefälligen Schulterklopfen unter Kirchenleuten, denn es sei nicht ausgeblendet, dass unsere Landeskirche in ihrer Quasi-Staatlichkeit ebenfalls einen beachtlichen Verwaltungsapparat geschaffen hat, der nicht frei von Erstarrungstendenzen und Überregulierung ist. Kommt hinzu, dass die schrumpfenden Einnahmen einer falsch verstandenen Vorstellung von diakonischer Effektivität Vorschub leisten. Umso wichtiger ist es daher, dass sich die Kirche an dem orientiert, was sie menschlich, lebendig, agil und flexibel macht und ausmacht. Die hier postulierte flexible Kirche und eine entsprechende diakonische Praxis ist denn auch nicht ein Befund, sondern als Ziel gedacht, das es beharrlich zu verfolgen in Abgrenzung zum Kirchen-Apparat zu verteidigen gilt.

\section{Zivilgesellschaft aktivieren und sozialraumorientiert han-} deln

Die Metapher von der Kirche, die im Dorf steht, ist reichlich abgedroschen. Sie sei hier doch nochmals bemüht, um aufzuzeigen, was die Volkskirche von vielen anderen Glaubens- und Wertegemeinschaften abhebt. Sie hat einen Ort und der Kirchturm ist auch heute meist noch sein auffälligstes Orientierungszeichen. Dieser Ort ist zumindest historisch als Sozialraum sowohl territorial als Kirchensprengel als auch sozial als Kirchgemeinde definiert. Man könnte sagen - um einen etwas angejahrten Begriff aus der Sozialen Arbeit zu verwenden - die typisch schweizerische
Jahrbuch Diakonie Schweiz 4 (2020/2021) http://dx.doi.org/10.22018/JDS.2021.14 
Kirchgemeinde adressiert ein Gemeinwesen und dieses ist grösser als die Zahl der reformierten Kirchensteuerzahlenden im betreffenden Einzugsgebiet und viel grösser als die der darin lebenden praktizierenden Reformierten.

Dieses Gemeinwesen formiert sich nicht nur durch die Summe einzelner Menschen, sondern konstituiert sich auch in den Einrichtungen und aus der Vielzahl weiterer Gemeinschaften. Die Kirche darf sich je nach örtlicher Gegebenheit mal bescheiden als eine dieser Gemeinschaften sehen und mal initiativ als deren Vernetzerin. Sie ist nicht die einzige, aber historisch wohl die wichtigste Akteurin und Mitgestalterin dessen, was wir heute Zivilgesellschaft nennen. Sie darf und soll als solche ihre Rolle selber definieren und sich Freiheiten herausnehmen, welche staatlichen Ämtern nicht zustehen. Menschen, die in ihr aktiv sind, dienen als «role model» für die heute vielbeschworenen ehrenamtlich Engagierten.

Es handelt sich hierbei um Freiwillige, die für im engeren Sinne kirchliche, aber auch weiter ausgreifende gesellschaftliche Aufgaben und Projekte arbeiten. Das Spektrum dafür ist weit und reicht von der Kirchenpflegerin bis zum Besucher im Altersheim. Es sind dies eben gerade nicht Menschen, welche die Sozialarbeiterin ersetzen (auch nicht den Diakon), sondern angeleitet durch die ausgebildeten Fachleute, aber mit möglichst hoher Eigenbestimmtheit Arbeiten ausführen, die sonst niemand im professionellen Sozialapparat übernimmt. Dieses freiwillige Engagement zu initiieren und $\mathrm{zu}$ orchestrieren ist ein wichtiges diakonisches Tätigkeitsfeld.

\section{Soziokulturelle Zentren bespielen, statt Kirchengut verwal-} ten

Nebst diesem Potential an motivierten Menschen eröffnen auch die zentral gelegenen Kirchengebäude sozialräumliche Chancenfelder. Diese Immobilien - der Begriff legt es schon nahe - werden allerdings angesichts hoher Unterhaltskosten aktuell eher als lähmende Klumpen am Fusse einer Kirche wahrgenommen, die sich in Bewegung setzen möchte. Zwar öffnen sich viele Kirchen als Orte der Kultur auch für nicht kultische Konzerte oder Theateraufführungen, doch findet diese Öffnung immer noch eher zögerlich statt.

Kirchgebäude und mit ihnen die oft grossen Gemeindehäuser könnten jedoch noch viel offensiver als bisher als eigentliche soziokulturelle Dorfoder Quartierzentren begriffen und genutzt werden. Dabei handelt es sich recht eigentlich um eine Nutzung im ursprünglichen Sinn, allerdings im Kontext einer neuen, sich zunehmend multireligiös, multikulturell und weltlich verstehenden Gesellschaft. Die nicht kirchlich betriebenen Gemeinschaftszentren in der Stadt Zürich mögen dafür ein Modell abgeben.

Solche Zentren gibt es längst nicht in allen Städten und wenn, dann nicht in derselben Dichte. Vor allem aber lässt sich deren Anzahl in den nichtstädtischen Gemeinden etwa im Kanton Zürich an einer Hand abzählen. Die Kirche könnte in diese Lücke springen. So würde sie ihren Besitz nicht bloss verwalten, sondern als soziokulturell ausgerichtete Gemeinschaftszentren, als Orte der Begegnung und des Austausches neu bewirtschaften und bespielen.

Diakoninnen und Diakone dürften sich darin auch als Animatorinnen und als Gemeinwesenarbeiter verstehen, die als Betriebspersonal in enger Zusammenarbeit mit Sigristinnen und Sigristen diesen Einrichtungen Leben einhauchen. Sie betreiben damit aktiven Gemeindeaufbau; einen Gemeindeaufbau allerdings, der sich nicht auf den Aufbau der reformierten Gemeinde fokussiert, sondern Menschen im ganzen Einzugsgebiet - und ohne den steten Hintergedanken der Mitgliederwerbung - anspricht. Zu hoffen wäre dabei, dass sich ein solches breites soziokulturelles Angebot auch die jeweilige politische Gemeinde etwas kosten liesse.

\section{Lebensthemen moderieren}

Das vierte Chancenfeld ragt über ein eng geführtes sozialarbeiterisches Selbstverständnis von Diakonie hinaus und mischt sich mit pfarrlichen Aufgabengebieten. Das entspricht durchaus dem, was gängige Praxis in den Gemeinden ist, nämlich die Mitarbeit im Rahmen von Gottesdiensten
Jahrbuch Diakonie Schweiz 4 (2020/2021) - ISSN 2504-3994

$$
\text { CC by } 4.0
$$

Jahrbuch Diakonie Schweiz 4 (2020/2021) http://dx.doi.org/10.22018/JDS.2021.14 
oder der Religionspädagogik. Allerdings lässt sich diese Nahtstelle zwischen den beiden kirchlichen Arbeitsfeldern auch unter einem nicht a priori konfessionsgebundenen theologischen Licht betrachten. Zwar schöpft das meiste, was Kirche im Kern ausmacht und im Laufe der Jahrhunderte entwickelt worden ist, selbstverständlich aus der Quelle des christlichen Glaubens; doch hat sich dabei auch ein «Handwerk» entwickelt, das für viele Menschen in verschiedenen Lebenslagen und jenseits ihrer Bekenntnisse oder Nichtbekenntnisse wertvoll sein kann.

Ganz generell ist die Kirche - bzw. ihr Personal - da, um Menschen in der Bewältigung von Lebensthemen und -aufgaben beizustehen. Das kann mal eher eine rituell-kultische oder mal eher eine seelsorgerische Form annehmen. Doch in beiden Fällen geht es je länger desto weniger darum, aus Bibelstellen wohlfeile Antworten und Ratschläge abzuleiten, sondern Menschen in ihren existentiellen Findungsprozessen zu begleiten und diese zu moderieren.

Seit jeher begleitet die Kirche nicht nur die Einzelnen in schwierigen Lebensphasen, sondern gestaltet deren lebensgeschichtlichen Initiationsund Übergangsphasen - Geburt und Aufgenommensein in der Gemeinschaft, Erwachsenwerden, Bindung, Abschied - und verleiht ihnen Form. Solche für die Einzelnen, für Familie, Verwandtschaft, Freundeskreis, ja für die Gemeinschaft wertvollen Inszenierungen und Ritualisierungen können vom kirchlichen Personal auch ohne Bezug zu Gott und Bibel und abseits einer im engen Sinn christlicher Liturgie vorgenommen werden. Es ist dies bereits gängige Praxis, wo Kirchenferne zum Beispiel eine kirchliche Abdankung für ihre Nächsten wünschen. Für dieses Zugeständnis brauchen sich denn auch weder die Pfarrerin noch der Diakon zu schämen. Ganz im Gegenteil; weshalb sollten sie mit ihrer Kompetenz nicht auch eine explizit konfessionsungebundene Feier gestalten helfen, ist dieser Beistand doch Ausdruck tätiger Nächstenliebe, die keine konfessionellen Schranken kennt?

Das genannte Beispiel verweist zwar stärker in die pfarrliche Domäne. Doch unter dem Gesichtspunkt fliessender Übergänge zwischen den Ar-

$$
\text { CC by } 4.0
$$

beitsfeldern ist dies auch für das diakonische Selbstverständnis von Belang, das eben gerade die weitere Rahmung der Sozialen Arbeit betont. Eine solche, flexible, empathische, den Klientinnen und Klienten zugewandte Soziale Arbeit rückt die Diakonie unweigerlich auch in die Nähe der Seelsorge und damit wieder zum Pfarrpersonal.

Doch gerade eine diakonische aufgefasste Sorge stellt das seelische Wohl im Hier und Jetzt ins Zentrum und ist eben nicht wie weiland auf das «Seelenheil» nach dem Tod fixiert. Diese Unterstützung darf sich durchaus - aber muss sich nicht zwingend - aus einem christlichen Glaubensbekenntnis speisen. Und vor allem kann sie ganz ohne Verkündigungen, Fürbitten, Bibelzitaten und Gesängen auskommen, wenn dies nicht dem Wunsch des Gegenübers entspricht. Mit einem Beispiel, soll hier dieser Gedanke aus einer anderen Perspektive illustriert werden: Die Europäerin, welche einen indischen Ernährungsberater aufsucht, muss sowenig dem christlichen Glauben abschwören und sich dem Hinduismus zuwenden, wie der Ayurveda-Experte die religiöse Verankerung seiner Kunst im Hinduismus verleugnen muss.

Es ist nicht so, dass die Kirche die in den Chancenfeldern skizzierten Angebote nie formuliert hätte. Doch wirkt sie dabei oft etwas gehemmt und von sich selber nicht ganz überzeugt; jedenfalls für noch zu viele Nicht-Reformierte zu wenig überzeugend. Vielleicht wäre es gar nicht so abwegig, sich die Kirche - um es mal in der nüchternen Sprache des Managements auszudrücken - als «Kompetenzzentrum für Anteilnahme, Lebensbegleitung und zur feierlichen Gestaltung von Übergängen» zu denken und sich als solches - nochmals ein Managementwort - selbstbewusst $\mathrm{zu}$ «positionieren». Zu verstehen ist dies als ein Akt der Sachlichkeit, nicht aber der emotionalen Kälte.

Die Kirche braucht nicht das Risiko zu fürchten, dass sie damit ihre Seele verliert. Vielmehr packt sie so die Chance, neu den Zugang zu einem ihrer durch religiöse Auseinandersetzungen oftmals zugeschütteten gesellschaftlichen Wesenskerne zu finden; nämlich dem der Hüterin und Pflegerin des gesellschaftlichen Zusammenhalts. Mehr noch: mit einer solchen mehr berufsethisch-handwerklichen denn theologischen Fundierung ihrer 
Arbeit findet die Kirche hoffentlich auch den Ausweg aus der drohenden Selbstblockierung einer minoritären Glaubensgemeinschaft, die am Anspruch festhält, in unserer vielfältigen Gesellschaft mit der Postulierung dieses Glaubens, statt mit den darauf beruhenden Taten als Volkskirche erkennbar für alle da zu sein. Die neben ihrer Glaubensverbundenheit ebenfalls vorhandene sehr lebensweltliche, diesseitige und praktisch ausgerichtete Tradition in der Diakonie könnte da ein verlässlicher Wegweiser zur offenen Kirche der Zukunft sein.

Autor:

Urs Frey, ehemaliger Co-Leiter des CAS Diakonie - Soziale Arbeit in der Kirche, ist sowobl Mitglied der Arbeitsgruppe Grundlagen und Forschung der Diakonie Schweiz als auch der stadtzürcherischen Kirchenkreiskommission sieben und acht 